\title{
The Behavioral Immune System (and Why It Matters)
}

\author{
Mark Schaller' and Justin H. Park ${ }^{2}$ \\ 'University of British Columbia and ${ }^{2}$ University of Bristol
}

\begin{abstract}
Like many other animals, human beings engage in behavioral defenses against infectious pathogens. The behavioral immune system consists of a suite of psychological mechanisms that (a) detect cues connoting the presence of infectious pathogens in the immediate environment, (b) trigger disease-relevant emotional and cognitive responses, and thus (c) facilitate behavioral avoidance of pathogen infection. However, the system responds to an overly general set of superficial cues, which can result in aversive responses to things (including people) that pose no actual threat of pathogen infection. In addition, the system is flexible, such that more strongly aversive responses occur under conditions in which perceivers are (or merely perceive themselves to be) more vulnerable to pathogen infection. Recent research reveals many provocative implications-for the experience of disgust, for extraversion and social interaction, for xenophobia and other prejudices, and for the origins of cultural differences.
\end{abstract}

\section{Keywords}

culture, disease, disgust, personality, prejudice, behavioral immune system

For a very long time and for many organisms, infectious diseases have posed a threat to reproductive fitness. As a consequence, there evolved sophisticated physiological mechanisms (the immune system) that detect and mobilize defenses against pathogens that enter the body. But it can be costly to actually mount an immune response. Therefore, there also evolved an additional set of mechanisms that serves as a crude first line of defense against pathogens - what can be called a behavioral immune system. These mechanisms detect the presence of pathogens in the immediate environment and facilitate the avoidance of those pathogens before they make contact with the body. Behavioral immune responses have been documented across many animal species (Kavaliers, Choleris, \& Pfaff, 2005; Kiesecker, Skelly, Beard, \& Preisser, 1999) and have important implications for human health, human psychology, and human culture.

\section{Basic Principles: Signal Detection and Functional Flexibility}

The behavioral immune system is sensitive to perceptual cues indicating that pathogens may be present. When perceived, these stimuli trigger adaptive psychological responsesincluding the arousal of specific kinds of aversive emotional experiences and the activation of specific kinds of cognitive knowledge structures in working memory-that facilitate behavioral avoidance.
These perceptual cues (e.g., noxious odors, disfiguring blemishes) are only imperfectly correlated with actual infection. Consequently, there emerges a signal-detection problem, and inference errors inevitably occur. Any strategic bias that limits the number of false-positive errors (erroneously inferring the presence of pathogens where there are none) leads to an increase in false-negative errors (erroneously inferring the absence of pathogens where, in fact, they exist), and vice versa. As with other systems designed for self-protection, this signaldetection problem is adaptively resolved in a manner defined by the "smoke detector principle" (Nesse, 2005). Household smoke detectors (the sort that you might install in your ceiling) are calibrated to minimize the likelihood of (potentially fatal) false-negative errors. As an inevitable consequence, they are sensitive to anything that superficially resembles smoke from a fire, and so are prone to make lots of (irritating but nonfatal) false-positive errors. Analogously, the behavioral immune system evolved to minimize the likelihood of (potentially fatal) false-negative errors. As a consequence, it is sensitive to cues that superficially resemble actual symptoms of pathogen infection and so is prone to make lots of false-positive errors.

\section{Corresponding Author:}

Mark Schaller, Department of Psychology, University of British Columbia, 2 I 36 West Mall, Vancouver BC V6T IZ4, Canada

E-mail: schaller@psych.ubc.ca 
The upshot is that threat of infection may be implicitly inferred from an overly inclusive set of superficial stimuli, including stimuli that are not actually symptoms of infection. This signal-detection bias has important implications that we discuss below.

In addition to the signal-detection problem, the behavioral avoidance of pathogens also poses a cost/benefit problem. While there are functional benefits associated with the detection and avoidance of things (including people) that superficially appear to pose some risk of infection, there are costs as well (e.g., calories consumed, opportunities missed). The relative ratio of costs to benefits is a function of an individual's vulnerability to infection. When individuals are relatively invulnerable to infection, the benefits offered by behavioral avoidance may be outweighed by its costs. But when individuals are highly vulnerable to infection, those costs may be outweighed by the benefits of behavioral avoidance. Accordingly, as with many other adaptive psychological systems, the behavioral immune system is characterized by the principle of functional flexibility (Schaller, Park, \& Kenrick, 2007): Under circumstances in which individuals are (or merely perceive themselves to be) especially vulnerable to pathogen infection, they are especially attentive to the presence of cues that indicate pathogens may be present, and these cues trigger especially strong aversive responses. This principle of functional flexibility has implications for a wide range of emotional, cognitive, and behavioral phenomena.

\section{Implications for Emotion (Specifically Disgust)}

Emotional experiences play an important role in the psychology of self-defense. Specific emotional experiences are triggered by the perception of specific kinds of threat-indicating cues, and these emotional experiences in turn facilitate engaging in functionally adaptive behaviors. There is a substantial body of research implicating the specific emotional experience of disgust as a key component of the behavioral immune system (Oaten, Stevenson, \& Case, 2009). The functional relationship between disease and disgust manifests in many ways. Sensory cues that most clearly connote proximity to infectious pathogens are especially likely to arouse disgust; disgust is also evoked by behaviors that violate normative expectations in behavioral domains that are functionally associated with disease transmission (e.g., food preparation, personal hygiene, sexual interaction; for a review, see Oaten et al., 2009). In addition, disgust responses appear to be calibrated to basic principles of contagion, such as the role of physical contact in the spread of disease (Rozin, Millman, \& Nemeroff, 1986).

Consistent with the smoke-detector principle, people experience disgust in response to an overly inclusive set of perceptual stimuli. People are disgusted not only by things that pose a real risk of pathogen infection (e.g., dog feces), they also are disgusted by things that pose no risk at all but that simply bear some superficial resemblance to real risks (e.g., chocolate fudge sculpted into the shape of dog feces; Rozin et al., 1986). Further, consistent with the principle of functional flexibility, people show more exaggerated responses to disgust-eliciting cues when the body's actual immunological defenses are temporarily suppressed (Fessler, Eng, \& Navarrete, 2005).

\section{Implications for Interpersonal Interaction}

Many diseases are transmitted as a result of interpersonal interaction. For this reason, while gregarious social behavior offers interpersonal benefits, it also connotes a specific kind of cost as well: increased likelihood of pathogen infection. For some people (e.g., individuals with chronically heightened sensitivity to disgust) the potential for infection may be chronically salient. For these individuals, the perceived costs associated with social interaction may be relatively high, and this has implications for interpersonal behavior. Consistent with the logic of this cost/benefit analysis, people who report chronically higher levels of "germ aversion" also report chronically lower levels of extraversion (Duncan, Schaller, \& Park, 2009). Analogously, the salience of potential infection may be temporarily heightened by specific circumstances (e.g., exposure to exaggerated media coverage of influenza outbreaks). These temporary circumstances can produce inhibitory effects on social interaction. In one set of experiments, participants were randomly assigned to either a control condition or a condition in which pathogens were made temporarily salient. Pathogen salience caused participants to report lower levels of extraversion, and to engage in avoidant motor movements when presented with pictures of people (Mortensen, Becker, Ackerman, Neuberg, \& Kenrick, 2010). The psychological salience of potential pathogen infection appears to cause a reduced inclination for social interaction.

\section{Implications for Person Perception and Prejudice}

While the psychological salience of potential infection may contribute to unsociable behavior in general, it also contributes to discriminatory unsociable behavior. It produces especially aversive responses to people who appear especially likely to pose some risk of pathogen transmission. This has implications for the stigmatization of people suffering from infectious diseases. For instance, prejudicial responses toward such people may be especially pronounced when perceivers feel especially vulnerable to infection. Plus, because of the smoke-detector principle, there is another pernicious implication as well: Psychologically similar prejudicial responses may be aroused by the perception of people who aren't actually suffering from any infectious disease but who are simply characterized by some superficial anomaly in physical appearance. And (because of the functional flexibility principle) these prejudices too may be especially pronounced when perceivers feel especially vulnerable to infection.

Consistent with this general hypothesis, a body of evidence now implicates the behavioral immune system as a contributing cause of prejudices against people whose physical appearance 


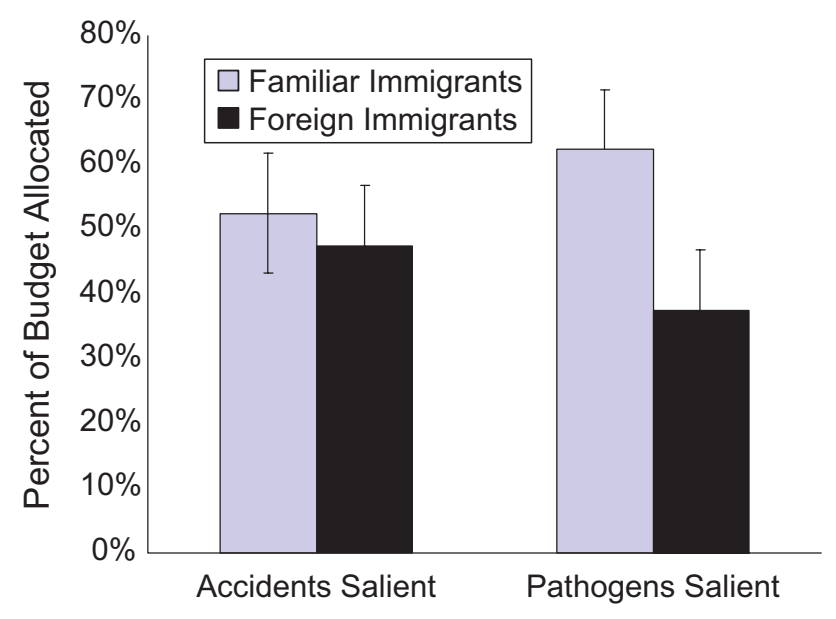

Fig. I. Immigration attitudes as predicted by perceived vulnerability to infectious diseases (Faulkner, Schaller, Park, \& Duncan, 2004, Study 6). Canadian participants were asked to allocate relative percentages of a government fund to recruit immigrants from various countries, some that were subjectively familiar (e.g., Poland, Taiwan) and others that were subjectively foreign (e.g., Mongolia, Peru). They did so after first watching one of two different slide shows. In a control condition, the slide show made salient the threat posed by disease-irrelevant accidents and mishaps (e.g., electrocution). In the other condition, the slide show made salient the threat posed by infectious pathogens. Results revealed that when pathogens were salient, participants were more inclined to spend government money recruiting immigrants from relatively familiar places, to the exclusion of those from places they considered foreign.

seems anomalous. The perceived threat of infection predicts more strongly prejudicial responses against people with physical disabilities, against people who are obese, and against people who are elderly (Duncan \& Schaller, 2009; Park, Faulkner, \& Schaller, 2003; Park, Schaller, \& Crandall, 2007). These findings illuminate a single subtle psychological process that contributes to a wide variety of appearance-based prejudices.

The behavioral immune system also contributes to ethnocentrism and xenophobia. Again, this implication results from findings documenting exaggerated prejudices among people who are (or merely perceive themselves to be) especially vulnerable to infectious diseases. One particularly provocative finding revealed that women in their first trimester of pregnancy - when the immune system is naturally suppressed - exhibited heightened ethnocentric and xenophobic attitudes (Navarrete, Fessler, $\&$ Eng, 2007). Another set of studies, employing a variety of methods, revealed that when people feel especially vulnerable to infection, they are especially likely to favor contact with familiar rather than foreign peoples (Faulkner, Schaller, Park, \& Duncan, 2004; see Fig. 1 for a detailed summary of results from one of these studies).

\section{Implications for Culture and Cross-Cultural Differences}

The principle of functional flexibility applies not only at an individual level of analysis but at a societal level of analysis as well. Under ecological circumstances in which there is greater prevalence of disease-causing pathogens, human populations are more likely to be characterized by behavioral norms that limit the likelihood of infection. An illustrative example is provided by the use of culinary spices. Most spices are natural antibiotics; their use in food preparation is a beneficial form of antipathogen defense. There are also costs associated with the cultivation and use of spices. The benefits are more likely to outweigh costs under ecological conditions of high pathogen prevalence. Accordingly, the use of culinary spices is more common under ecological circumstances characterized by high pathogen prevalence (Sherman \& Billing, 1999). The same logic applies to specific kinds of psychological traits, attitudes, and values. To the extent that some dispositional tendency puts people at risk for pathogen infection, that dispositional tendency is likely to vary across human populations, depending on the prevalence of pathogens in the local ecology. This has implications for the prediction of many cross-cultural differences of considerable interest to psychological scientists.

Consider extraversion once again. As discussed above, extraversion is associated with social benefits but also with disease-specific costs. Those costs are especially pronounced under ecological circumstances of high pathogen prevalence. Therefore, in countries characterized by relatively higher prevalence of pathogens, the population is likely to be characterized by relatively lower levels of extraversion. Schaller and Murray (2008) reported multiple tests of this hypothesis, based on multiple measures of personality collected from thousands of people in dozens of countries worldwide. Consistent with the hypothesis, higher levels of pathogen prevalence were associated with lower levels of extraversion.

A similar logical analysis can be applied to another fundamental dimension of personality: openness to experience. Openness implies curiosity, risk-taking, and a willingness to deviate from cultural norms (some of which, especially in realms such as hygiene and food preparation, inhibit pathogen transmission). These dispositional tendencies are likely to increase exposure to pathogens - especially under conditions of high pathogen prevalence. Therefore, in countries characterized by relatively higher prevalence of pathogens, the population is likely to be characterized by relatively lower levels of openness. This is exactly what worldwide personality data reveal (Schaller \& Murray, 2008).

This kind of analysis can be extended into the realm of cultural value systems as well-including the individualism/ collectivism dimension that is so fundamental to the psychological study of cultural differences. Collectivism is defined in part by an emphasis on conformity to existing cultural traditions and norms, whereas individualism is defined in part by a tolerance for (and even encouragement of) deviance. Therefore, individualistic values are associated with increased risk of pathogen infection, especially under conditions of high pathogen prevalence. The logical implication is that collectivistic value systems are especially likely to emerge and persist under ecological circumstances characterized by high pathogen prevalence, whereas individualistic value systems are more 


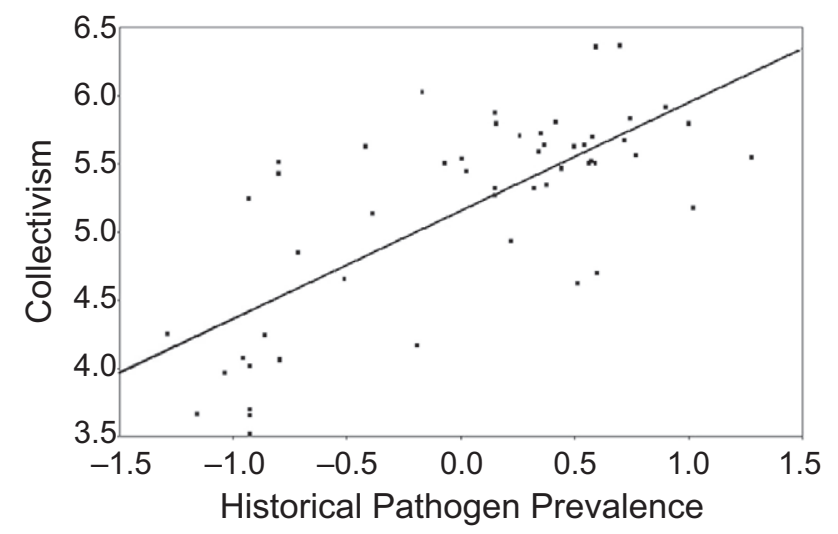

Fig. 2. Cross-cultural variation in collectivistic values as predicted by worldwide ecological variation in pathogen prevalence $(r=.73$; Fincher, Thornhill, Murray, \& Schaller, 2008). This figure is representative of an effect replicated across multiple measures of individualism/ collectivism: In countries with historically higher levels of pathogen prevalence (standardized scores), contemporary cultural value systems are more strongly collectivistic. Correlations remained significant even when controlling for plausible confounding variables.

likely to emerge and persist under ecological circumstances characterized by relatively lower pathogen prevalence. Worldwide data on individualism and collectivism support this hypothesis (Fincher, Thornhill, Murray, \& Schaller, 2008; see Fig. 2).

These and other cross-cultural findings - on outcomes ranging from sexual behavior (Schaller \& Murray, 2008) to social liberalism and political democratization (Thornhill, Fincher, \& Aran, 2009) - suggest that many important cross-cultural differences may owe their existence, in part, to long-standing ecological differences in the relative prevalence of pathogens.

\section{Future Directions}

These different lines of inquiry attest to the generative power of a few simple logical principles that have governed research on human behavioral defenses against pathogens. They also attest to the important contribution that myriad superficially different phenomena (e.g., emotional responses, cognitive appraisals, behavioral prejudices, cultural norms) contribute to antipathogen defense.

These findings also give rise to a whole new set of research questions that remain unanswered. Consider the intriguing relation between pathogen prevalence and cultural differences. While it appears increasingly apparent that there may be a causal link between pathogen prevalence and cultural differences, the exact mechanisms that account for that link have not yet been identified. A variety of different mechanisms are plausible, operating at different levels of analysis (e.g., differential genetic selection, differential developmental expression of common genes, differential activation of functionally flexible neurocognitive mechanisms, differential cultural transmission of learned behaviors). Careful elucidation of these underlying mechanisms is an important direction for future research.
Other exciting directions for future research pertain more directly to human health outcomes. Because the behavioral immune system appears to have implications for social gregariousness, it is likely also to influence the size, shape, and geometry of social networks. This has important epidemiological implications that remain unexplored. There is also new evidence that mere visual perception of diseased-looking people can trigger perceivers' own white blood cells to mount a more vigorous response to bacterial infection (Schaller, Miller, Gervais, Yager, \& Chen, 2010). The intriguing implication, which also demands further investigation, is that activation of the behavioral immune system might affect functioning of the real immune system too.

\section{Recommended Reading}

Gangestad, S.W., Haselton, M.G., \& Buss, D.M. (2006). Evolutionary foundations of cultural variation: Evoked culture and mate preferences. Psychological Inquiry, 17, 75-95. Summarizes evidence linking pathogen prevalence to cross-cultural differences in mate preferences, and also explains how local ecological conditions, in conjunction with universal human tendencies, may create cross-cultural differences; also accompanied by a wide-ranging set of commentaries.

Oaten, M., Stevenson, R.J., \& Case, T.I. (2009). (See References). A comprehensive review of research on disgust and the role it plays in the avoidance of infectious diseases.

Schaller, M., \& Duncan. L.A. (2007). The behavioral immune system: Its evolution and social psychological implications. In J.P. Forgas, M.G. Haselton, \& W. von Hippel (Eds.), Evolution and the social mind: Evolutionary psychology and social cognition (pp. 293-307). New York, NY: Psychology Press. An accessible introduction to the behavioral immune system and how it works, accompanied by an illustrative review of evidence linking these mechanisms to person perception and prejudice.

Schaller, M., \& Murray, D.R. (2008). (See References). A detailed presentation of evidence linking pathogen prevalence to crosscultural differences in personality; includes also a discussion of different mechanisms that may account for these effects.

\section{Declaration of Conflicting Interests}

The authors declared that they had no conflicts of interest with respect to their authorship or the publication of this article.

\section{Funding}

Preparation of this article was facilitated by a research grant from the Social Sciences and Humanities Research Council of Canada.

\section{References}

Duncan, L.A., \& Schaller, M. (2009). Prejudicial attitudes toward older adults may be exaggerated when people feel vulnerable to infectious disease: Evidence and implications. Analyses of Social Issues and Public Policy, 9, 97-115.

Duncan, L.A., Schaller, M., \& Park, J.H. (2009). Perceived vulnerability to disease: Development and validation of a 15 -item self-report instrument. Personality and Individual Differences, 47, 541-546. 
Faulkner, J., Schaller, M., Park, J.H., \& Duncan, L.A. (2004). Evolved disease-avoidance mechanisms and contemporary xenophobic attitudes. Group Processes and Intergroup Behavior, 7, 333-353.

Fessler, D.M.T., Eng, S.J., \& Navarrete, C.D. (2005). Elevated disgust sensitivity in the first trimester of pregnancy: Evidence supporting the compensatory prophylaxis hypothesis. Evolution and Human Behavior, 26, 344-351.

Fincher, C.L., Thornhill, R., Murray, D.R., \& Schaller, M. (2008). Pathogen prevalence predicts human cross-cultural variability in individualism/collectivism. Proceedings of the Royal Society B, 275, 1279-1285.

Kavaliers, M., Choleris, E., \& Pfaff, D. W. (2005). Recognition and avoidance of the odors of parasitized conspecifics and predators: Differential genomic correlates. Neuroscience \& Biobehavioral Reviews, 29, 1347-1359.

Kiesecker, J.M., Skelly, D.K., Beard, K.H., \& Preisser, E. (1999). Behavioral reduction of infection risk. Proceedings of the National Academy of Sciences, 96, 9165-9168.

Navarrete, C.D., Fessler, D.M.T., \& Eng, S.J. (2007). Elevated ethnocentrism in the first trimester of pregnancy. Evolution and Human Behavior, 28, 60-65.

Nesse, R.M. (2005). Natural selection and the regulation of defenses: A signal detection analysis of the smoke detector principle. Evolution and Human Behavior, 26, 88-105.

Mortensen, C.R., Becker, D.V., Ackerman, J.M., Neuberg, S.L., \& Kenrick, D.T. (2010). Infection breeds reticence: The effects of disease salience on self-perceptions of personality and behavioral tendencies. Psychological Science, 21, 440-447.
Oaten, M., Stevenson, R.J., \& Case, T.I. (2009). Disgust as a diseaseavoidance mechanism. Psychological Bulletin, 135, 303-321.

Park, J.H., Faulkner, J., \& Schaller, M. (2003). Evolved diseaseavoidance processes and contemporary anti-social behavior: Prejudicial attitudes and avoidance of people with physical disabilities. Journal of Nonverbal Behavior, 27, 65-87.

Park, J.H., Schaller, M., \& Crandall, C.S. (2007). Pathogen-avoidance mechanisms and the stigmatization of obese people. Evolution and Human Behavior, 28, 410-414.

Rozin, P., Millman, L., \& Nemeroff, C. (1986). Operation of the laws of sympathetic magic in disgust and other domains. Journal of Personality and Social Psychology, 50, 703-712.

Schaller, M., Miller, G.E., Gervais, W.M., Yager, S., \& Chen, E. (2010). Mere visual perception of other people's disease symptoms facilitates a more aggressive immune response. Psychological Science, 21, 649-652.

Schaller, M., \& Murray, D.R. (2008). Pathogens, personality, and culture: Disease prevalence predicts worldwide variability in sociosexuality, extraversion, and openness to experience. Journal of Personality and Social Psychology, 95, 212-221.

Schaller, M., Park, J.H., \& Kenrick, D T. (2007). Human evolution and social cognition. In R.I.M. Dunbar \& L. Barrett (Eds.), Oxford handbook of evolutionary psychology. (pp. 491-504). Oxford, England: Oxford University Press.

Sherman, P.W., \& Billing, J. (1999). Darwinian gastronomy: Why we use spices. BioScience, 49, 453-463.

Thornhill, R., Fincher, C.L., \& Aran, D. (2009). Parasites, democratization, and the liberalization of values across contemporary countries. Biological Reviews, 84, 113-131. 